\title{
A Price-and-Branch algorithm for a drayage problem with heterogeneous trucks
}

\author{
Ali Ghezelsoflu, Massimo Di Francesco, Paola Zuddas ${ }^{1}$ \\ Department of Mathematics and Computer Science, University of Cagliari, \\ Cagliari, Italy \\ Antonio Frangioni ${ }^{2}$ \\ Department of Computer Science, University of Pisa, Pisa, Italy
}

\begin{abstract}
This paper investigates a drayage problem, which is motivated by a carrier providing door-to-door freight transportation services by trucks and containers. The trucks carry one or two containers to ship container loads from a port to importers and from exporters to the same port. The problem is modeled by a set covering formulation with integer variables. We propose a Price-and-Branch algorithm for this problem, in which the pricing problem is a pair of shortest path problems in a suitable graph. The algorithm can determine near-optimal solutions in a short time.
\end{abstract}

Keywords: Price-and-Branch, Drayage, Vehicle Routing Problem, Set Covering.

\section{Introduction}

In this problem a carrier manages a fleet of trucks to serve two types of customer requests: the delivery of container loads from a port to importers and the shipment of container loads from exporters to the same port. Some trucks carry one container and can serve up to one importer and one exporter in a route. Other trucks can carry up to two containers and can serve up to two

1 Email: ali.nov7@gmail.com, mdifrance@unica.it, zuddas@unica.it

2 Email: frangio@di.unipi.it 
importers and two exporters in a route. Customers must be serviced according to a stay-with policy, i.e. drivers wait for containers during packing and unpacking operations and trucks carry the same containers throughout their routes. Each truck performs only one route, routing costs between any pair of locations depends only on their distances and the truck type, as two-containers trucks have higher costs per unitary distance than one-container trucks.

This problem has several original characteristics. Trucks were usually supposed to carry one container at a time and the distribution of more-than-one container per truck has received attention only in recent papers (e.g., (4)). Moreover, in many drayage problems trucks are decoupled from containers at customer locations and trucks can perform different tasks during packing and unpacking operations (see (1) for a detailed literature literature). Few papers investigated both multiple containers per truck and stay-with operations as in our problem $(2 ; 3)$. These papers proposed node-arc formulations, which have very poor linear relaxations. A set-covering formulation for this problem was proposed by (1), where all feasible routes were enumerated and an off-theshelf optimization solver was run to optimally solve realistic-sized instances. However, it runs out-of-memory in the case of large-scale problems.

This paper proposes a Price-and-Branch algorithm for the formulation of (1). In the first step, the linear programming relaxation of the set-covering problem is solved by column generation, where the pricing problem is a shortest path problem in a proper graph. The resulting fractional optimal solution value is a tight lower bound on the value of the optimal integer solution. Then, from the set of columns generated so far, an integer solution is sought by Cplex. The computational results are compared to those in (1).

The paper is organized as follows. In Section 2, the set mathematical model is presented. In section 3 the Price-and-Branch algorithm is proposed. In Section 4, the results of the computational experiments are presented. Finally, conclusions and further research perspectives are described in Section 5.

\section{Modeling}

Let $I$ be the set of importers, $E$ the set of exporters and $V=I \cup E$ the set of customers, let $R_{1}$ and $R_{2}$ be the set of all feasible routes performed by one-container and two-container trucks, respectively, and $R=R_{1} \cup R_{2}$ the set of all feasible routes. More precisely, $R_{1}$ and $R_{2}$ are the sets of routes leaving from the port, serving up to two and four customers respectively and moving back to the same port. If $n=\max \{|E|,|I|\}$, the number of all the possible routes is $O\left(n^{4}\right)$. Moreover, we denote by $k_{1}$ and $k_{2}$ the number of available 
trucks for one-container and two-containers routes, respectively

For each route $r \in R$ and each customer $v \in V$, define the coefficient $\alpha_{r}^{v}$ such that $\alpha_{r}^{v}=0$ if customer $v \in V$ is not visited in route $r \in R, \alpha_{r}^{v}=1$ if customer $v \in V$ is visited in route $r \in R$ to deliver or pick up one container load, $\alpha_{r}^{v}=2$ if customer $v \in V$ is visited in route $r \in R_{2}$ to deliver or pick up two container loads. Let $d_{v}$ be the demand of each customer $v \in V$, i.e., the number of containers which must be used to service customer $v$. The decision variable $x_{r}$ is defined as the number of times in which route $r \in R$ is performed, each time paying the corresponding unitary $\operatorname{cost} c_{r}$. According to this notation, the problem can be formulated as follows:

$$
\begin{array}{rlr}
Z_{P}=\min & \sum_{r \in R} c_{r} x_{r} & \\
\text { s.t. } & \sum_{r \in R} \alpha_{r}^{v} x_{r} \geq d_{v} & v \in V \\
& \sum_{r \in R_{1}} x_{r} \leq k_{1} & \\
& \sum_{r \in R_{2}} x_{r} \leq k_{2} & \\
& x_{r} \in \mathbb{Z}_{+} & r \in R
\end{array}
$$

Routing costs are minimized in the objective function (1). Constraints (2) ensure that all customers are served. Constraints (3) and (4) enforce that the number of routes is lower than the number of available corresponding trucks. Finally, constraint (5) defines the domain of decision variables.

\section{Price-and-Branch algorithm}

To solve the problem effectively, a Price-and-Branch algorithm is proposed. A restricted set of all possible routes is enumerated and the linear relaxation with this partial route set (or Restricted Master Problem [RMP]) is solved. Let $B_{1} \subseteq R_{1}$ and $B_{2} \subseteq R_{2}$ be the subsets of routes with one-container and two-containers trucks, respectively, where $B=B_{1} \cup B_{2}$ and $B \subseteq R$. The RMP is formulated as follows:

$$
\begin{array}{rlr}
z_{R M P}=\min & \sum_{r \in B} c_{r} x_{r} & \\
\text { s.t. } & \sum_{r \in B} \alpha_{r}^{v} x_{r} \geq d_{v} & v \in V \\
& \sum_{r \in B_{1}} x_{r} \leq k_{1} & \\
& \sum_{r \in B_{2}} x_{r} \leq k_{2} & \\
& x_{r} \in \mathbb{R}_{+} & r \in B
\end{array}
$$

The optimal solution of the RMP may be sub-optimal for the master problem with all feasible routes. However, the values of the optimal dual variables 
of the RMP can be used to identify if there are any routes not included in the RMP that can further reduce the objective function value $z_{R M P}$. Let $\xi_{v}, \pi$, and $\varpi$ multipliers of constraints (7), (8), and (9) for each $v \in V$. The dual of the RMP is denoted by DMP and is formulated as follows:

$$
\begin{array}{rlr}
z_{D M P}=\max & \sum_{v \in V} \xi_{v} d_{v}-\pi k_{1}-\varpi k_{2} & \\
\text { s.t. } & \sum_{v \in V} \xi_{v} \alpha_{r}^{v}-\pi-\varpi \leq c_{r} & r \in B \\
& \xi_{v} \in \mathbb{R}_{+} & v \in V \\
& \pi, \varpi \in \mathbb{R}_{+} &
\end{array}
$$

Let $x^{*}$ be the optimal solution of RPM and $\xi^{*}, \pi^{*}, \varpi^{*}$ the optimal solution of DMP. Although this solution satisfies constraint (12), there may exist at least a route $r \in R \backslash B$ violating this constraint. If so, this route is added to $B$, the RMP is solved again to determine a better value of $z_{R M P}$ and so on until no violated constraint is found.

Determining these violated constraints means looking for columns with negative reduced costs, where the reduced cost of route $r$ is $\left\{c_{r}^{*}=c_{r}-\left(\sum_{v \in V} \xi_{v}^{*} \alpha_{r}^{v}-\right.\right.$ $\left.\left.\pi^{*}-\varpi^{*}\right)\right\}$. These columns are determined in the pricing problem, where one minimizes $c_{r}^{*}$, such that capacity constraints are met.

The key-point of this Price-and-Branch algorithm is that the pricing problem can be formulated as a pair of shortest path problems on the following acyclic graph, one for each the truck type.

- In the case of one-container trucks, the port is split into two nodes $p$ and $p^{\prime}$ and each customer $v \in V$ is modeled by one node. Arcs link $p$ to any customer $v \in V$, any importer to any exporter, any customer $v \in V$ to $p^{\prime}$. Each arc is associated with a unitary routing cost.

- In the case of two-container trucks, the port is again split into two nodes $p$ and $p^{\prime}$ and each customer is modeled by two nodes $v^{\prime} \in V^{\prime}$ and $v^{\prime \prime} \in V^{\prime \prime}$. Arcs link $p$ to any node $v^{\prime} \in V^{\prime}$, any pair of nodes $v^{\prime} \in V^{\prime}$ and $v^{\prime \prime} \in V^{\prime \prime}$ associated with each customer $v \in V$, any importer $i \in V^{\prime \prime}$ to any exporter $e \in V^{\prime}$ and vice versa, any node $v^{\prime} \in V^{\prime}$ and $v^{\prime \prime} \in V^{\prime \prime}$ to $p^{\prime}$. Each arc connecting two different locations is associated with a unitary routing cost, whereas all arcs from $v^{\prime} \in V^{\prime}$ to $v^{\prime \prime} \in V^{\prime \prime}$ have a null cost.

Fig. 1 represents the acyclic graphs in the case of two importers denoted by 1 and 2, and three exporters denoted by 3, 4 and 5 .

In the new graph, the pricing problem is a pair of two shortest path problems from $p$ to $p^{\prime}$ : the first determines a route for one-container trucks, the second a route for two-container trucks. If both solutions are negative, add 


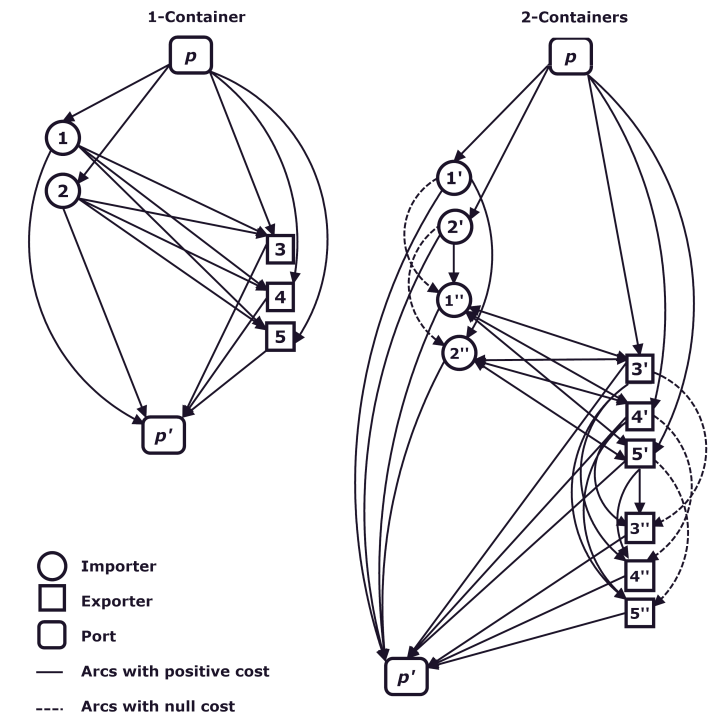

Fig. 1. Acyclic graph with five customers for one-container and two-containers trucks

both routes to the RMP. If only one of them is negative, add it to the RMP. The column generation stops when both routes determined in the pricing have a nonnegative reduced cost. If so, solve a RMP with the updated set $B$ in which the decision variables are required to be integer.

\section{Computational tests}

Computational tests are carried out on some instances taken from (2). The RMP and the pricing problems in Section 3 has been solved by Cplex 12.5 on a Linux server with $3.00 \mathrm{Ghz}$ processor, $16 \mathrm{~GB}$ of RAM. The restricted set of routes is initialized with all direct trips made by one-container and two-container trucks. The outcomes are reported in Tables 1 . The following notation is used:

- it and $\left|B^{*}\right|$ are the number of iterations of the column generation algorithm and the number of columns of the RMP at the end of the column generation;

- $t_{P B}, t_{M}, t_{P}$ and $t_{l m}$ are times in seconds to solve the Price-and-Branch algorithm, the column generation algorithm, the linear relaxation of master problem at the end of the column generation, the integer master problem at the end of the column generation;

- $\tau_{n}$ and $t_{n}$ are the preprocessing time in seconds to generate all $|R|$ feasible 
routes for the set-covering model and the time in seconds to solve the setcovering model by Cplex, respectively;

- Gap 1 is the relative gap between the upper bound determined by the integer RMP in Price-and-Branch algorithm and the optimal solutions of the setcovering formulation with all feasible routes;

- Gap2 is the relative gap between the optimal solutions of set-covering formulation and the linear relaxation of the RMP at the end of Price-and-Branch algorithm.

The results in Table 1 show the effectiveness of the Price-and-Branch algorithm. First, $\left|B^{*}\right|$ is significantly lower than $|R|$, thus it takes a short time to determine integer solutions from the RMP. Nevertheless, the solutions of the algorithm Price-and-Branch are near-optimal. Second, the time of the Price-and-Branch is a bit larger than sum of the overall time to enumerate all feasible routes and solve the set covering model in the case of all instances with 10 and 20 customers, but it is faster in almost of instances with 40 and 50 customers. Therefore, it is of interest to test if this trend holds event in the case of larger instances. They are taken from (1) and the related computational tests are reported in Table 2, where the same notation of Table 1 is adopted. The results in Table 2 show that the Price-and-Branch algorithm can determine near-optimal solutions for all the instances. The set covering with all feasible routes takes a much longer running time to solve instances $\mathrm{H}$, I and J. Moreover, it does not return any feasible solution in the case of instances K, L, M, and N, as Cplex runs out-of-memory. 


\begin{tabular}{|c|c|c|c|c|c|c|c|c|c|c|c|c|c|c|}
\hline \multirow[b]{2}{*}{$|I|$} & \multirow[b]{2}{*}{$|E|$} & \multirow[b]{2}{*}{$k_{1}$} & \multirow[b]{2}{*}{$k_{2}$} & \multicolumn{6}{|c|}{ Price-and-Branch } & \multicolumn{3}{|c|}{ Enumeration } & & \\
\hline & & & & $i t$ & $\left|B^{*}\right|$ & $t_{P B}$ & $t_{M}$ & $t_{P}$ & $t_{l m}$ & $R$ & $\tau_{n}$ & $t_{n}$ & Gap1 & Gap2 \\
\hline 2 & 8 & 2 & 9 & 14 & 40 & 0.575 & 0.554 & 0.008 & 0.077 & 462 & 0.02 & 0.09 & 0.003 & 0.100 \\
\hline 5 & 5 & $\overline{2}$ & 7 & 14 & 42 & 0.482 & 0.457 & 0.008 & 0.062 & 810 & 0.10 & 0.17 & 0.005 & 0.093 \\
\hline 8 & 2 & 5 & 9 & 13 & 40 & 0.455 & 0.434 & 0.007 & 0.070 & 366 & 0.03 & 0.07 & 0.004 & 0.114 \\
\hline 2 & 8 & 0 & 10 & 14 & 40 & 0.658 & 0.637 & 0.007 & 0.053 & 462 & 0.02 & 0.09 & 0.014 & 0.120 \\
\hline 5 & 5 & 0 & 8 & 14 & 42 & 0.603 & 0.577 & 0.007 & 0.041 & 810 & 0.10 & 0.17 & 0.024 & 0.076 \\
\hline 8 & 2 & 0 & 12 & 13 & 40 & 0.572 & 0.547 & 0.008 & 0.046 & 366 & 0.03 & 0.07 & 0.005 & 0.105 \\
\hline 2 & 18 & 8 & 22 & 24 & 81 & 1.129 & 1.071 & 0.036 & 0.062 & 1792 & 0.05 & 0.27 & 0.006 & 0.066 \\
\hline 5 & 15 & 7 & 19 & 30 & 86 & 1.383 & 1.284 & 0.049 & 0.159 & 7095 & 0.16 & 0.40 & .023 & 0.070 \\
\hline 10 & 10 & 5 & 14 & 31 & 84 & 1.474 & 1.396 & 0.050 & 0.054 & 11220 & 0.34 & 0.28 & 0.016 & 0.066 \\
\hline 15 & 5 & 7 & 19 & 28 & 83 & 1.544 & 1.412 & 0.047 & 0.216 & 6345 & 0.26 & 1.75 & 0.005 & 0.072 \\
\hline 18 & 2 & 5 & 24 & 26 & 81 & 1.199 & 1.132 & 0.042 & 0.058 & 1716 & 0.05 & 0.31 & 0.009 & 0.052 \\
\hline 2 & 18 & 0 & 26 & 24 & 81 & 1.115 & 1.052 & 0.035 & 0.081 & 1792 & 0.05 & 0.35 & 0.004 & 0.056 \\
\hline 5 & 15 & 0 & 23 & 30 & 86 & 1.498 & 1.405 & 0.053 & 0.068 & 7095 & 0.16 & 0.46 & 0.006 & 0.041 \\
\hline 10 & 10 & 0 & 17 & 31 & 84 & 2.403 & 2.331 & 0.048 & 1.186 & 11220 & 0.35 & 0.61 & 0.000 & 0.051 \\
\hline 15 & 5 & 0 & 23 & 28 & 83 & 1.585 & 1.500 & 0.050 & 0.134 & 6345 & 0.26 & 0.94 & 0.014 & 0.063 \\
\hline 18 & 2 & 0 & 27 & 26 & 81 & 1.442 & 1.377 & 0.040 & 0.184 & 1716 & 0.05 & 0.37 & 0.004 & 0.057 \\
\hline 2 & 28 & 13 & 33 & 39 & 124 & 1.986 & 1.819 & 0.118 & 0.102 & 5578 & 0.29 & 0.26 & 0.002 & 0.069 \\
\hline 5 & 25 & 12 & 30 & 42 & 124 & 2.117 & 1.966 & 0.115 & 0.137 & 19555 & 1.4 & 1.12 & .007 & 0.072 \\
\hline 10 & 20 & 10 & 25 & 46 & 127 & 3.215 & 3.007 & 0.157 & 0.147 & 44730 & 1.72 & 4.39 & 0.028 & 0.045 \\
\hline 15 & 15 & 8 & 19 & 54 & 143 & 3.542 & 3.336 & 0.152 & 0.313 & 54480 & & 3.48 & 0.021 & 0.051 \\
\hline 20 & 10 & 10 & 26 & 48 & 131 & 2.746 & 2.563 & 0.136 & 0.072 & 42730 & 2.15 & 4.27 & 0.002 & 0.070 \\
\hline 25 & 5 & 12 & 32 & 38 & 122 & 1.788 & 1.655 & 0.100 & 0.065 & 17055 & 1.49 & 1.29 & 0.008 & 0.060 \\
\hline 28 & 2 & 14 & 35 & 33 & 119 & 1.607 & 1.491 & 0.087 & 0.089 & 4122 & 0.83 & 0.28 & 0.004 & 0.059 \\
\hline 2 & 28 & 0 & 40 & 39 & 124 & 2.19 & 2.043 & 0.117 & 0.187 & 5578 & & 0.44 & 0.032 & 0.040 \\
\hline 5 & 25 & 0 & 36 & 42 & 124 & 2.313 & 2.151 & 0.123 & 0.077 & 19555 & 1.49 & 3.08 & 0.030 & 0.049 \\
\hline 10 & 20 & 0 & 30 & 46 & 127 & 2.521 & 2.297 & 0.168 & 0.216 & 44730 & 1.72 & 6.31 & 0.020 & 0.053 \\
\hline 15 & 15 & 0 & 23 & 54 & 143 & 3.090 & 2.908 & 0.135 & 0.057 & 54480 & 2.11 & 6.72 & 0.014 & 0.058 \\
\hline 20 & 10 & 0 & 31 & 48 & 131 & 3.2 & 3.004 & 0.153 & 0.052 & 42730 & 2 . & 9.62 & 0.011 & 0.063 \\
\hline 25 & 5 & 0 & 38 & 38 & 122 & 2.260 & 2.116 & 0.101 & 0.116 & 17055 & 1.38 & 4.10 & 0.025 & 0.043 \\
\hline 28 & 2 & 0 & 42 & 33 & 119 & 1.870 & 1.749 & 0.093 & 0.079 & 4122 & 0.83 & 0.28 & 0.025 & 0.030 \\
\hline 2 & 38 & 20 & 49 & 44 & 160 & 2.230 & 1.988 & 0.201 & 0.138 & 10228 & 0.09 & 1.68 & 0.067 & 0.031 \\
\hline 5 & 35 & 18 & 45 & 49 & 161 & 2.578 & 2.293 & .237 & 107 & 38215 & 1.72 & 2.34 & 020 & 0.066 \\
\hline 10 & 30 & 14 & 38 & 57 & 165 & 4.0 & 3.754 & 0.274 & 0.106 & 100340 & 4.19 & 5.37 & 047 & 0.032 \\
\hline 15 & 25 & 12 & 31 & 60 & 171 & 4.481 & 4.011 & 0.351 & .144 & 1265 & 6.8 & 7.11 & 0.034 & 0.048 \\
\hline 20 & 20 & 12 & 29 & 69 & 179 & 4.448 & 3.998 & 0.377 & 0.098 & 168840 & 8.3 & 18.4 & 0.030 & 0.062 \\
\hline 25 & 15 & 14 & 36 & 59 & 160 & 3.855 & 3.533 & 0.233 & 0.062 & 147515 & 6.39 & 7.47 & 0.029 & 0.072 \\
\hline 30 & 10 & 17 & 43 & 54 & 164 & 3.1 & 2.859 & 0.228 & & 94340 & 4.01 & 5.27 & 0.028 & 0.061 \\
\hline 35 & 5 & 19 & 48 & 45 & 159 & 3.2 & 3.026 & 0.221 & 31 & 32965 & 1.5 & 2.12 & 0.028 & 0.061 \\
\hline 38 & 2 & 20 & 51 & 45 & 162 & 2.462 & 2.228 & 0.188 & 0.208 & 7492 & 0.09 & 0.81 & 23 & 0.062 \\
\hline 2 & 38 & 0 & 59 & 44 & 160 & 2.964 & 2.720 & 0.200 & 0.187 & 10228 & 0.09 & 5.38 & 0.069 & 0.019 \\
\hline 5 & 35 & 0 & 54 & 49 & 161 & 2.9 & 2.712 & 0.225 & 0.291 & 38215 & 1.72 & 3.41 & 0.070 & 0.025 \\
\hline 10 & 30 & 0 & 45 & 57 & 165 & 4.5 & 4.236 & 0.297 & & & 4. & 8 & & .020 \\
\hline 15 & 25 & 0 & 37 & 60 & 171 & 5.718 & 5.255 & 0.390 & 0.498 & 151265 & 6.83 & 7.89 & 0.081 & 0.021 \\
\hline 20 & 20 & 0 & 35 & 69 & 179 & 5.252 & 4.794 & 0.388 & 0.126 & 3840 & 8.35 & 18.5 & 0.070 & 0.022 \\
\hline 25 & 15 & 0 & 43 & 59 & 165 & 4.451 & 4.018 & 0.367 & 0.605 & 147515 & 6.3 & 7.52 & 0.068 & 0.021 \\
\hline 30 & 10 & 0 & 51 & 54 & & 3.3 & & & & 4340 & & & & 0.023 \\
\hline 35 & 5 & 0 & 58 & 45 & 159 & 2.850 & 2.597 & 0.207 & 0.132 & 32965 & 1.51 & 1.68 & 0.070 & 0.018 \\
\hline 38 & 2 & 0 & 61 & 45 & 162 & 2.669 & 2.402 & 0.189 & 0.188 & 7492 & 0.09 & 1.14 & 0.066 & 0.019 \\
\hline 2 & 48 & 22 & 56 & 56 & & & 2.757 & & & & & & & \\
\hline 5 & 45 & 21 & 54 & 62 & 202 & 3.796 & 3.286 & 0.449 & & 62850 & 4.19 & 1.67 & 33 & 069 \\
\hline 10 & 40 & 18 & 50 & 70 & 202 & 5.6 & 4.9 & & & & & & & .034 \\
\hline 15 & 35 & 17 & 42 & 70 & 201 & 5.1 & 4.411 & & & & 17 & 14.0 & 0.073 & 0.038 \\
\hline 20 & 30 & 13 & 37 & 80 & 210 & 7.0 & 6.250 & 0.6 & 0.0 & & 23 & 23.2 & 0.073 & 0.038 \\
\hline 25 & 25 & 11 & 32 & 77 & 218 & 4.702 & 4.133 & 0.492 & 0.094 & 7550 & 24.7 & 16.2 & 0.071 & 0.035 \\
\hline 30 & 20 & 12 & 32 & 78 & 219 & & 4.334 & & & & 22.2 & 16.2 & & 0.034 \\
\hline 35 & 15 & 15 & 39 & 73 & 206 & 4.4 & 3.936 & & & & & 13.0 & & 0.031 \\
\hline 40 & 10 & 17 & 46 & 73 & 211 & 4.5 & 4.010 & 0.4 & 0.109 & 750 & 9.96 & 4.61 & 85 & 0.039 \\
\hline 45 & 5 & 20 & 50 & 62 & 205 & 3.872 & 3.389 & 0.405 & 0.123 & 53850 & 3.39 & 2.01 & 0.064 & 0.035 \\
\hline 48 & 2 & 22 & 55 & 55 & 201 & 3.393 & 2.955 & 0.381 & & & 0.73 & 1.14 & 0.049 & 0.035 \\
\hline 2 & 48 & 0 & 67 & 56 & 203 & 3.941 & 3.464 & & & & & 1.17 & & 0.015 \\
\hline 5 & 45 & 0 & 65 & 62 & 202 & 4.072 & 3.607 & 0.395 & 0.151 & 62850 & 4.19 & 2.71 & 0.095 & 0.015 \\
\hline 10 & 40 & 0 & 59 & 70 & 202 & 5.599 & 4.970 & 0.540 & 0.266 & 177750 & 11. & 5.11 & 99 & 0.015 \\
\hline 15 & 35 & 0 & 51 & 70 & 201 & 7.258 & 6.335 & 0.709 & 1.431 & 295500 & 17.9 & 14.9 & 0.100 & 0.020 \\
\hline 20 & 30 & 0 & 44 & 80 & 210 & 7.99 & 7.150 & 0.737 & 0.1 & & 23.1 & 13.2 & & 0.024 \\
\hline 25 & 25 & 0 & 38 & 77 & 218 & 5.144 & 4.591 & 0.480 & & 407550 & 24.7 & 17.4 & 0.094 & 0.021 \\
\hline 30 & 20 & 0 & 38 & 78 & 219 & 5.4 & 4.870 & 0.470 & 0.0 & 373350 & 22 & 16.7 & 0.122 & 0.015 \\
\hline 35 & 15 & 0 & 47 & 73 & 206 & 4.856 & 4.368 & 0.420 & 0.259 & 285000 & 17.4 & 7.92 & 0.122 & 0.019 \\
\hline 40 & 10 & 0 & 55 & 73 & 211 & 4.931 & 4.439 & 0.430 & 0.121 & 165750 & 9.96 & 3.81 & 0.118 & 0.014 \\
\hline 45 & 5 & 0 & 60 & 62 & 205 & 4.490 & 4.046 & 0.383 & 0.166 & 53850 & 3. & 1.48 & & 0.020 \\
\hline 48 & 2 & 0 & 66 & 55 & 201 & 3.803 & 3.346 & 0.399 & 0.142 & 11862 & 0.73 & 1.23 & 0.085 & 0.019 \\
\hline
\end{tabular}

\section{Table 1}

Comparison on realistic-sized instances between the Price-and-Branch algorithm and the set-covering formulation enumerating all feasible routes (1) 


\begin{tabular}{|c|c|c|c|c|c|c|c|c|c|c|c|c|c|}
\hline & \multirow[b]{2}{*}{$|I|$} & \multirow[b]{2}{*}{$|E|$} & \multicolumn{6}{|c|}{ Price-and-Branch } & \multicolumn{3}{|c|}{ Enumeration } & \multirow[b]{2}{*}{ Gap1 } & \multirow[b]{2}{*}{ Gap2 } \\
\hline & & & $i t$ & $\left|B^{*}\right|$ & $t_{P B}$ & $t_{M}$ & $t_{P}$ & $t_{l m}$ & $R$ & $\tau_{n}$ & $t_{n}$ & & \\
\hline A & 20 & 5 & 28 & 59 & 1.149 & 1.093 & 0.043 & 0.133 & $1.1 \mathrm{e}+4$ & 1.01 & 0.22 & 0.00025 & 0.00036 \\
\hline B & 20 & 10 & 47 & 93 & 2.051 & 1.907 & 0.112 & 0.059 & $4.3 \mathrm{e}+4$ & 1.94 & 1.19 & 0.00061 & 0.00045 \\
\hline $\mathrm{C}$ & 20 & 20 & 62 & 136 & 2.971 & 2.691 & 0.236 & 0.048 & $1.7 \mathrm{e}+5$ & 8.98 & 4.00 & 0.00026 & 0.00024 \\
\hline $\mathrm{D}$ & 30 & 8 & 46 & 94 & 2.060 & 1.877 & 0.155 & 0.245 & $6.1 \mathrm{e}+4$ & 5.03 & 9.09 & 0.00021 & 0.00024 \\
\hline $\mathrm{E}$ & 30 & 15 & 67 & 132 & 3.449 & 3.084 & 0.319 & 0.130 & $2.1 \mathrm{e}+5$ & 6.83 & 13.3 & 0.00041 & 0.00028 \\
\hline $\mathrm{F}$ & 30 & 30 & 103 & 206 & 6.028 & 5.139 & 0.811 & 0.059 & $8.4 \mathrm{e}+5$ & 15.7 & 20.3 & 0.00084 & 0.00091 \\
\hline $\mathrm{G}$ & 45 & 12 & 67 & 136 & 3.439 & 2.900 & 0.488 & 0.160 & $3.0 \mathrm{e}+5$ & 8.65 & 16.3 & 0.00034 & 0.00017 \\
\hline $\mathrm{H}$ & 45 & 23 & 95 & 194 & 6.283 & 5.292 & 0.922 & 0.805 & $1.1 \mathrm{e}+6$ & 13.06 & 2455.7 & 0.00029 & 0.00019 \\
\hline I & 45 & 45 & 148 & 300 & 11.73 & 9.184 & 2.434 & 0.092 & $4.2 \mathrm{e}+6$ & 30.48 & 782.9 & 0.00037 & 0.00024 \\
\hline $\mathrm{J}$ & 75 & 19 & 115 & 230 & 8.137 & 6.025 & 2.028 & 0.642 & $2.1 \mathrm{e}+6$ & 18.49 & 5560.3 & 0.00034 & 0.00023 \\
\hline $\mathrm{K}$ & 75 & 38 & 177 & 340 & 16.02 & 11.46 & 4.422 & 0.138 & $8.2 \mathrm{e}+6$ & 49.25 & n.s. & - & - \\
\hline $\mathrm{L}$ & 75 & 75 & 275 & 556 & 34.55 & 22.34 & 11.86 & 0.115 & $3.2 \mathrm{e}+7$ & 157.29 & n.s. & - & - \\
\hline $\mathrm{M}$ & 100 & 25 & 162 & 313 & 15.04 & 10.06 & 4.837 & 0.619 & $6.3 e+6$ & 38.14 & n.s. & - & - \\
\hline $\mathrm{N}$ & 100 & 50 & 215 & 425 & 24.53 & 15.38 & 8.940 & 0.988 & $2.5 \mathrm{e}+7$ & 107.32 & n.s. & - & - \\
\hline
\end{tabular}

Table 2

Comparison on larger instances

\section{Conclusion}

In this paper we have investigated a drayage problem faced by a carrier. We have proposed a Price-and-Branch algorithm, in which a pair of shortest path problems is solved at each iteration of the pricing problem. The algorithm can determine near-optimal solutions in short time intervals and is very useful to solve large instances efficiently. An extension of this research will be the development of a Branch-and-Price algorithm to determine optimal solutions.

\section{References}

[1] Ghezelsoflu, A. and Di Francesco, M. and Frangioni, A. and Zuddas, P., A Set-Covering formulation for a drayage problem with single and double container loads, Journal of Industrial Engineering International, DOI (2018), 10.1007/s40092-018-0256-8.

[2] Lai, M. and Crainic, T. G. and Di Francesco, M. and Zuddas, P., An heuristic search for the routing of heterogeneous trucks with single and double container loads, Transportation Research Part E: Logistics and Transportation Review 56, (2013), 108-118.

[3] Lai, M. and Battarra, M. and Di Francesco, M. and Zuddas, P., An adaptive guidance meta-heuristic for the vehicle routing problem with splits and clustered backhauls, Journal of the Operational Research Society 66, (2015), 1222-1236.

[4] Zhang, R. and Yun, W.Y. and Kopfer, H., Multi-size container transportation by truck: modeling and optimization, Flexible Services \& Manufacturing 27, (2015), 403-430. 